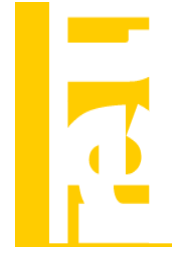

\title{
SEGMENTACIÓN LABORAL Y CONTRATACIÓN DUAL: CONVERSACIONES PÓSTUMAS CON LUIS TOHARIA
}

\author{
Virginia Hernanz*, Juan F. Jimeno ${ }^{\dagger}$ \\ *Universidad de Alcalá \\ †Banco de España
}

\section{Resumen}

Este artículo revisa la investigación de Luis Toharia relacionada con la segmentación del mercado de trabajo. Se estudian tanto los conceptos teóricos que utilizó (basados en la definición de segmentación de puestos de trabajo de Doeringer-Piore) como su análisis del caso español sobre las diferencias entre trabajadores con contratos temporales y con contratos indefinidos.

Palabras clave: Segmentación, contratos temporales, contratos indefinidos.

Clasificación JEL: J20, J21, J23, J41.

\begin{abstract}
This article reviews the Luis Toharia research on labour market segmentation. The survey focuses on his theoretical analyses (based on the jobs' segmentation definition proposed by Doeringer and Piore) and his analyses about the differences between workers with temporary and open-ended contracts.

Key words: Segmentation, temporary contracts, open-ended contracts.
\end{abstract}

JEL Classification: J20, J21, J23, J41.

\section{Introducción}

El análisis de la segmentación laboral es un tema recurrente en Economía Laboral. Desde cualquier aproximación a la realidad del mercado de trabajo, resulta evidente que los puestos de trabajo difieren en muchas características (salario, estabilidad, beneficios no pecuniarios, posibilidades de promoción, etc.), diferencias que se traducen en la existencia de "buenos" y "malos" puestos de trabajo. Determinados grupos de trabajadores están sobrerrepresentados entre los puestos de trabajo de "mayor calidad", mientras que los puestos de trabajo de "menor calidad" son ocupados con mayor frecuencia por grupos de trabajadores con diferentes características individuales. Entender las causas que

(c) Revista de Economía Laboral 
determinan la composición de puestos de trabajo por "calidades", el acceso de los trabajadores a los "buenos" y "malos" puestos de trabajo, y sus consecuencias en términos de desempleo y desigualdad social, no solo mejora la comprensión del funcionamiento del mercado de trabajo, sino que permite desarrollar políticas de empleo que aumenten el bienestar de los trabajadores.

En las primeras teorías sobre segmentación laboral (véase, por ejemplo, Doringer y Piore, 1971), eran los condicionantes tecnológicos y las pautas de demanda, con un notable componente sectorial, las principales fuerzas que determinaban la creación de puestos de trabajo de distinta naturaleza. Dichas teorías apuntaban, además, tres ideas que acabaron conformando la llamada visión "institucionalista" del mercado de trabajo: i) los "buenos" puestos de trabajo, pertenecientes al llamado mercado "primario" de trabajo, tienen asociados todas las características deseables (productividad elevada, salarios altos, beneficios no pecuniarios, estabilidad y están asociados a una carrera profesional atractiva con elevadas posibilidades de promoción) mientras que los "malos" (los que pertenecen al segmento "secundario" del mercado de trabajo) suelen serlo también en todas las dimensiones posibles, ii) los "buenos" puestos de trabajo escasean, por lo que no todos los trabajadores que tienen el nivel educativo adecuado y la cualificación suficiente para cubrir estos puestos de trabajo, acaban accediendo a uno de ellos, y iii) una elevada proporción de los "buenos" puestos de trabajo se cubren en mercados internos, es decir, tras acceder a la empresa que los ofrece por otros puestos de trabajo (llamados "puertos de entrada") desde los que se promociona al "mercado primario". Los mercados internos de trabajo se contemplan, según esta teoría (véanse, por ejemplo, Toharia, 1983; Doringer y Piore, 1971, Willianson et al., 1975) como el resultado del propio funcionamiento no competitivo del mercado de trabajo y de las estrategias optimizadoras de los empresarios que tratan de reducir costes de rotación laboral en ese contexto.

Más recientemente, a medida que las reformas laborales en Europa han introducido contratos de trabajo "no regulares", alternativos al contrato permanente, a tiempo completo con elevada protección al empleo y protegido por el poder negociador sindical en la negociación colectiva, sobre el que han pivotado las relaciones laborales desde mediados del siglo pasado, se ha generado una nueva forma de segmentación laboral, generada por la utilización de trabajadores con contratos distintos para la realización de tareas similares. Esta estrategia de reforma se ha justificado como una manera de promover el empleo de los trabajadores menos favorecidos en el mercado de trabajo y la flexibilidad empresarial a la hora de adaptarse a cambios tecnológicos o de 
demanda. Como consecuencia, de una visión "institucionalista" de la segmentación laboral se ha pasado a perspectivas más "neoclásicas" de esta cuestión, en las que, basándose en modelos que distinguen entre trabajadores "insiders" (protegidos por "contratos regulares") y "outsiders" (desempleados o trabajadores con contratos "atípicos"), se destacan las consecuencias de la segmentación contractual con relación al nivel y persistencia del desempleo, los salarios reales, la acumulación de capital humano y la viabilidad política de las reformas laborales (véase, por ejemplo, Saint-Paul, 1996).

Entre otras muchas, dos de las contribuciones de Luis Toharia al desarrollo del análisis económico del mercado de trabajo, fueron, en primer lugar, desarrollar y difundir la visión institucionalista del mercado de trabajo en España y, en segundo lugar, estudiar las consecuencias de la segmentación contractual para proponer mejoras en las políticas de empleo. Como coautores suyos en algunos trabajos sobre estos temas, tuvimos el privilegio de discutir con él muchas de las cuestiones que se suscitan sobre segmentación laboral y contratación dual. El objetivo de este artículo es revisar algunas de sus contribuciones en este campo y de sus ideas sobre la contratación laboral, tratando de enmarcar sus puntos de vista en el debate actual sobre reformas laborales y políticas de empleo. Para ello, lo hemos estructurado alrededor de tres preguntas fundamentales cuyas respuestas determinan en gran medida qué políticas de empleo pueden mejorar la configuración institucional del mercado de trabajo y, así, reducir el desempleo y la desigualdad. Estas tres preguntas son:

- ¿Qué factores determinan la segmentación del mercado de trabajo?

- ¿Qué consecuencias socioeconómicas tiene dicha segmentación?

- ¿En qué medida la contratación dual agrava la segmentación

laboral y sus consecuencias?

\section{2. ¿Qué factores determinan la segmentación del mercado de trabajo?}

La idea de el mercado de trabajo no funciona de forma competitiva ha existido en la literatura sobre las relaciones industriales al menos desde de la década de 1950, pero no es hasta la década de los años 1970s cuando aparecen un conjunto de autores (Doringer y Piore, 1971, y Williamson et al, 1975) que dotan a dicha idea de algo más de contenido señalando la segmentación como una de las características fundamentales 
del mercado laboral. Originariamente, el objetivo de estos desarrollos teóricos, que pronto se conocerían como "visión institucionalista" del mercado de trabajo era desarrollar una teoría de la inflación salarial, al margen de la determinación neoclásica de salarios en función de la oferta y la demanda de trabajo y en la que el desempleo no era un factor determinante de los salarios.

Casi al mismo tiempo, originada en una visión marxista de las relaciones laborales, se desarrolla lo que en Toharia (1981) se denomina la "teoría de la eficiencia", según la cual, el problema fundamental de los empresarios radica en convertir al fuerza de trabajo disponible en los empleados en trabajo efectivo (Bowles y Gintis, 1975). Desde esta visión marxista se critica la Economía Laboral neoclásica con el argumento de que esta se basa en una visión individualista que convierte el trabajo en capital y a los trabajadores en capitalistas, que hace irrelevante la distinción entre que los trabajadores sean contratados o que sean ellos los que alquilen el capital, y que elimina del análisis la importancia del control de los medios de producción.

La distinción entre fuerza de trabajo y trabajo, incorporada después al campo neoclásico, por ejemplo, en la teoría de los salarios de eficiencia, en el campo institucionalista sirve como fundamentación de los "mercados internos" de trabajo, entendidos como un complejo entramado de reglas y procesos administrativos que prometen a los trabajadores una carrera estable, fomentan la estabilidad en el empleo y permiten a las empresas sacar el máximo beneficio a su inversión inicial en la formación del trabajador. Toharia (1981) realiza un extenso repaso de estos modelos con mercados internos de trabajo y trata de distinguir entre dos corrientes dentro de esta teoría de la eficiencia, en función de los mecanismos: i) el poder de monopolio (Williamson et al.,1975) que surge cuando se requiere capital humano específico, asociado a cada puesto de trabajo, cuyo aprendizaje necesita de unos costes iniciales, para el desarrollo de "tareas indiosincráticas" que no pueden ser fácilmente satisfechas mediante la contratación de trabajadores en el mercado externo, y ii) los costes de rotación (Doringer y Piore, 1971), que incluyen costes de sustitución de trabajadores, también ligados a la existencia de cualificaciones específicas, y precio de terminación de la relación laboral

En definitiva, en esta visión institucionalista, la segmentación laboral aparece como resultado de la existencia de tareas idiosincráticas y de la respuesta de las empresas al problema de la eficiencia y de la rotación laboral. Así, acaban siendo la tecnología, en la medida en qué esta determina hasta qué punto son necesarias dichas tareas idiosincráticas, y las fluctuaciones estacionales o cíclicas de la demanda de productos, qué determinan las pautas de sustitución de trabajadores, los dos factores que 
subyacen a la segmentación del mercado de trabajo. La respuesta óptima de las empresas a tales condiciones tecnológicas y de demanda es la configuración de segmentos primarios y secundarios del mercado de trabajo y de los mercados internos de trabajo como principal mecanismo de contratación y de selección de trabajadores para el mercado primario. Por otra parte, la selección misma de tecnología es un instrumento de las empresas para resolver los problemas de eficiencia y rotación (Toharia, 1981). Además, el problema de la existencia de un segmento secundario no se resolvería incrementando la cualificación de la oferta de trabajo, pues aunque se ayudara a los trabajadores a adquirir las características de los del sector primario, por mucho que se eleve su capital humano, siempre existirán puestos de trabajo poco deseables en el mercado secundario, que dan lugar a los abandonos y a la elevada rotación laboral (Toharia, 1983). Así, se concluye que la existencia de "malos" puestos de trabajo es inevitable por ser una consecuencia imprescindible del desarrollo tecnológico y de la sociedad industrial.

Pero aparte de los condicionantes tecnológicos, las empresas, a la hora de tomar sus decisiones sobre creación y destrucción de puestos de trabajo, tienen en cuenta todos los componentes de los costes laborales, incluidos los asociados a la contratación y al despido. Es a través de este vía por la que la legislación laboral puede afectar tanto a la cantidad como a la composición del stock de puestos de trabajo existentes en cada momento. Este efecto es todavía más relevante cuando dicha legislación laboral establece condiciones diferentes para la creación y la destrucción de empleo en función de las características del puesto de trabajo, diferenciación que ha estado presente, de una manera o de otra y con una incidencia importante, en nuestra legislación laboral desde que en 1984 se liberalizó el uso de los contratos laborales. Primero, el contrato temporal de fomento del empleo, vigente hasta 1992, permitió la contratación durante un máximo de tres años, por periodos de seis meses, con un coste muy pequeño asociado a la no renovación del contrato durante ese periodo. Después, con la reintroducción, más aparente que real, del llamado "principio de causalidad", según el cual los contratos temporales solo podrían utilizarse para puestos de trabajo de duración determinada y conocida previamente a la realización del contrato, la incidencia de los contratos temporales ha seguido siendo elevada y diferenciando las condiciones de contratación y despido, y, por tanto, muchas otras condiciones de empleo, entre los nuevos entrantes en el mercado de trabajo y los que ya disponían de un contrato indefinido de trabajo.

Resulta un tanto irónico que desde la visión institucionalista del mercado de trabajo no se la haya prestado mucha atención a las consecuencias sobre la segmentación laboral de las instituciones laborales, 
entendidas estas como el conjunto de reglas, normas, contratos, construcciones sociales o entidades que regulan el mercado de trabajo y que no pueden ser cambiados rápida o fácilmente. ${ }^{1}$ Una justificación de tal omisión puede ser que, en su origen y como en tantos otros casos, la visión institucionalista se refería a mercados de trabajo anglosajones en los que la legislación laboral resulta menos vinculante. Sin embargo, Luis Toharia, que nunca dejó de creer en la predominancia de la visión institucionalista a la hora de analizar la segmentación laboral, sí entendió desde muy temprano, casi inmediatamente después de la liberalización de los contratos temporales en 1984, que esta "innovación" suponía un cambio radical en el funcionamiento del mercado de trabajo español. Desde entonces dedicó buena parte de su tiempo a documentar y entender dicho cambio y a tratar de formular políticas de empleo que trataran de aumentar los beneficios y disminuir los costes de la segmentación laboral.

\section{3. ¿Qué consecuencias socioeconómicas tiene la segmentación laboral?}

La segmentación laboral tiene obviamente consecuencias inmediatas sobre la desigualdad social. En la medida en que trabajadores de determinadas características sean más proclives a ocupar "malos" puestos de trabajo, de menor productividad y estabilidad, serán estos trabajadores los que tengan menores ingresos salariales y ocurrencias más frecuentes y duraderas de paro, menores posibilidades de promoción laboral y, en definitiva, peores condiciones laborales. Si la segmentación laboral responde a la visión institucionalista de distinción entre mercados primario y secundario, otra consecuencia es que la tasa de desempleo, al contrario de lo que postula la teoría neoclásica, no juega ningún papel como determinante de la fijación salarial, puesto que el desempleo ocurrirá fundamentalmente entre los trabajadores del mercado secundario, mientras que los del primario estarían protegidos por sus cualidades específicas que les llevaron a esa situación.

\footnotetext{
${ }^{1}$ Una señal reveladora de esta omisión es que durante una conferencia reciente de Michael Piore organizada por la Fundación Ortega y Gasset y celebrada en el Consejo Económico y Social en 2011, preguntado directamente el conferenciante sobre en qué medida las características de la legislación española sobre protección al empleo podrían condicionar la segmentación laboral, la respuesta fue bastante vaga y elusiva. Es también irónico que, en esto, los institucionalistas coinciden con los neoclásicos de la llamada Escuela de Chicago que tampoco prestan demasiada atención a las instituciones laborales en sus análisis del mercado de trabajo.
} 
Cuando a la segmentación laboral institucionalista se le añade la derivada de una legislación laboral dual, hay muchos aspectos de las condiciones laborales que pueden verse afectados. Por ejemplo, uno de los temas recurrentes en los análisis de Luis Toharia sobre el mercado de trabajo español es el estudio de las consecuencias de la segmentación laboral y, en particular, cómo la utilización de la flexibilidad en el margen proporcionada por los contratos temporales, pueden tener consecuencias tanto microeconómicas como macroeconómicas. Las características de los puestos pueden variar ya que la redefinición de los procesos productivos, sustentados en esta segmentación, no solo puede afectar a la especialización productiva de la economía, a las decisiones de inversión en formación, a las tasas de siniestralidad laboral y ha determinado el uso estratégico del sistema de prestaciones, sino que, además, puede tener consecuencias sobre la productividad agregada de la economía, sobre la tasa de desempleo y sobre el proceso de formación salarial.

En una de sus primeras líneas de investigación sobre este tema, Luis Toharia retoma lo originalmente expuesto en Doringer y Piore (1971), donde la necesidad de adquirir aprendizaje para habilidades específicas es uno de los factores que condiciona la segmentación entre distintos puestos de trabajo. Así, el uso tan intensivo de los contratos temporales, en la medida en qué reduce los incentivos a la formación específica, tanto a los empleadores como a los trabajadores, habría aumentado las dificultades para los trabajadores temporales para acceder al mercado primario. En España, tres de cada cuatro trabajadores temporales declara haber aceptado un contrato temporal por no haber podido acceder a un contrato indefinido, esta proporción es muy superior a otros países como es el caso de Italia, donde esta proporción se reduce a cuatro de cada diez (Hernanz et al, 2009). Estos trabajadores, además, presentan una menor incidencia de las actividades formativas (Albert et al, 2005). En concentro, según estos autores, los trabajadores temporales no sólo tienen una menor probabilidad de estar trabajando en empresas que den formación, sino que incluso cuando están trabajando en aquellas empresas que ofrecen formación a sus trabajadores, presentan una menor probabilidad de ser elegidos para participar en la misma.

Este impacto sobre las decisiones de inversión en capital humano influiría de forma directa no sólo sobre su productividad presente sino también a la productividad en el largo plazo (Andrés et al., 2009, Madera, 2012 , entre otros). Jimeno y Toharia (1996), sin embargo, analizaron las diferencias en la probabilidad de absentismo laboral entre los trabajadores indefinidos y temporales, obteniendo que aquellos trabajadores con contrato temporal muestran, una vez descontados los efectos composición y la influencia de enfermedades y accidentes profesionales, un menor 
absentismo. Es decir, la probabilidad de perder el empleo en el futuro reciente podría actuar como un incentivo para estos trabajadores. Trabajos más recientes, como el de Dolado y Stuchi (2008), también enfatizan el papel de la tasa de conversión de trabajadores temporales en indefinidos a la hora de proveer los incentivos adecuados para el aumento de la productividad. Según estos últimos autores, la bajísima tasa de conversión a contratos indefinidos en España podría explicar una parte relevante del escaso dinamismo de la productividad. En esta misma línea, Madera (2012) analiza el papel que juegan las diferencias en las políticas de protección al empleo, los costes de despido y el grado de rigidez con el que se regulan los contratos temporales para explicar las diferencias de productividad observadas en once países europeos y Estados Unidos. Sus resultados apuntan a que la presencia de un mercado segmentado tiene un impacto negativo sobre el crecimiento de la productividad de trabajo, aunque el impacto sobre la productividad total de los factores no es significativo

Las consecuencias de los contratos temporales son, en todo caso, más amplias. Por ejemplo, Hernanz y Toharia (2006) observaron que la probabilidad de tener un accidente laboral es superior si el trabajador ocupa un puesto de carácter temporal. Esta mayor siniestralidad de los trabajadores temporales esta fundamentalmente relacionada con la mayor presencia de trabajadores temporales en actividades más arriesgadas pues desaparece cuando se descuentan debidamente la influencia de las variables personales y del puesto de trabajo. Algo similar ocurre cuando se analiza la menor remuneración salarial de los trabajadores temporales. (Davia y Hernanz, 2004). Estas diferencias vienen dadas, en su mayor parte, por las fuertes diferencias que existen en las características de los trabajadores y, sobre todo, de los puestos de trabajo que ocupan.

Otros estudios han intentado analizar el impacto de la elevada temporalidad sobre factores como la emancipación de los jóvenes (Garrido et al, 1996) y la formación de nuevos hogares (Gutierrez-Domenech, 2008) y en general sobre su percepción del mercado de trabajo (Toharia, Davia y Hernanz, 2001). Los contratos temporales se han convertido en la principal vía de acceso para los individuos que se incorporan al mercado de trabajo. Las carreras laborales han sufrido una profunda transformación debido al uso intensivo de las formas más flexibles de empleo para la contratación de los más jóvenes. Se ha extendido el periodo durante el cuál los jóvenes experimentan unas trayectorias laborales más inestables y caóticas. Si bien, en Toharia (2005), se puso de manifiesto que a largo plazo no todos los jóvenes se ven necesariamente atrapados en la temporalidad. En este trabajo, Luis Toharia mostró, a partir de un análisis de cohortes "ficticias", utilizando los datos disponibles en la 
Encuesta de Población Activa (EPA), una pauta general de integración a medio/largo plazo en el empleo estable que se mantiene en todas las cohortes. No obstante, su análisis también mostró un porcentaje significativo de personas que parecen quedar atrapadas en la temporalidad, incluso cuando llegan a edades bastante maduras, en torno a los 40 años. Este análisis de cohortes se complementó en Toharia et al (2007) utilizando los datos de la Muestra Continua de Vidas Laborales que permite realizar un seguimiento exhaustivo del historial laboral de los trabajadores. Los resultados tienden a mostrar, de nuevo, como un colectivo de trabajadores, aunque no mayoritario, mantiene una situación de temporalidad durante un período de tiempo relativamente elevado. En concreto, por ejemplo, en torno al $20 \%$ de los trabajadores que firmaron un contrato temporal en el año 2000 se encontraban en esa situación a finales de 2004 .

\section{4. ¿En qué medida la contratación dual agrava la segmentación laboral y sus consecuencias socioeconómicas?}

Sobre esta cuestión, la posición de Luis Toharia era bastante clara $^{2}$. Aún reconociendo la importancia de la contratación dual para entender el funcionamiento del mercado de trabajo español, siempre se mantuvo firme en sus creencias sobre la importancia de la tecnología, el capital humano y, sobre todo, la gestión de los recursos humanos por las empresas como determinantes últimos de la segmentación laboral. A este respecto, no consideraba a la legislación laboral ni, en concreto, a la amplitud del menú de contratos de trabajo disponibles como factores muy relevantes. De hecho, utilizando las diferencias de la incidencia de la temporalidad entre regiones, sostuvo que eran las empresas las que, con una misma legislación, utilizaban los contratos temporales de forma muy diferente. Así, por ejemplo, Toharia (2005) concluye que la liberalización de contratos temporales en 1984, que permitió el uso no causal de los contratos temporales, no habría generado la estructura segmentada del mercado de trabajo español, que ya era un mercado que reposaba en una

\footnotetext{
${ }^{2}$ En sus propias palabras, tomadas de su último manuscrito, (e.g. Toharia, 2009) "El mercado de trabajo ni crea ni destruye empleo, lo que hace es transmitir al empleo la evolución de los mercados de productos. Ese "mecanismo de transmisión" puede funcionar mejor o peor, desde luego, pero para saberlo no basta con mirar a los resultados del empleo y el paro sino que hay que ver de dónde vienen las perturbaciones y si el mercado de trabajo está transmitiendo correcta o incorrectamente esas perturbaciones".
} 
estructura económica dualizada con puestos proclives a la estabilidad y otros de carácter inestables. Lo que sí habría permitido la disponibilidad de contratos temporales fue abrir una nueva vía de entrada en el mercado de trabajo, que ha sido utilizado por las empresas españolas como el principal instrumento de flexibilización y de ajuste del mercado de trabajo español ante todo tipo de variaciones en la producción. Han sido las empresas las que han acabado sobreutilizando estos contratos y reordenando los procesos productivos a partir de esta sobreutilización y, en muchas casos, lo habrían hecho con decisiones subóptimas en un contexto de "racionalidad limitada". ${ }^{3}$

Por tanto, según la visión que Luis Toharia siempre mantuvo, la segmentación del mercado de trabajo español se debe, en mayor medida, a factores relacionados con la demanda de trabajo, más que a las características institucionales del mercado laboral en España y, en concreto, a la existencia misma de contratos temporales. ${ }^{4} \mathrm{El}$ hecho de que la temporalidad incida más en determinados sectores, como la construcción, la agricultura y la hostelería, y que se concentre en determinados colectivos, especialmente los menos cualificados, eran para él prueba de esta tesis. Así, Toharia (2004), argumenta que las persistentes, e incluso divergentes, diferencias regionales, también apuntan en la dirección de la tesis mencionada, dadas las instituciones laborales comunes entre regiones. A este respecto, fue él también quién acuñó el término de la "cultura de la temporalidad" para explicar su alta incidencia en nuestro país, término que posteriormente otros han utilizado frecuentemente y de forma errónea en debates sobre reformas de la contratación laboral. Como corolario, , dado que el uso de la temporalidad en España respondería, en buena medida, a una lógica de maximización de los beneficios de los empresarios, las reformas encaminadas a eliminar legalmente los contratos temporales o a fomentar su conversión en contratos indefinidos correrían el riesgo de estar condenadas al fracaso (Toharia, 2005). Si los empresarios necesitan este tipo de contratos, para cubrir una parte muy relevante de su demanda, buscarían la vía para

\footnotetext{
${ }^{3}$ Luis Toharia sostenía que después de más de dos décadas de uso de estos contratos, estos se han convertido en una vía de contratación mayoritaria, basada en la "tradición/creencia/cultura" de que son la vía más eficaz para reducir los costes de rotación. Y sus posibles ventajas iniciales (Boeri et al 2007), ligadas a la reducción de los costes de rotación, habrían desparecido con el uso continuado y desvirtuado de los mismos.

4 En Toharia (1983) se señala que el único elemento del estudio de la demanda de trabajo que es verdaderamente propio del mercado de trabajo (es decir que hace que la demanda de trabajo no sea meramente una demanda derivada) proviene del concepto de capital humano.
} 
seguir utilizándolos o para alcanzar arreglos contractuales equivalentes. No obstante, también reconoce que la posible eliminación de las diferencias entre los trabajadores temporales y los indefinidos, a saber, que la igualación de los procedimientos extintivos y de las indemnizaciones por despido, tampoco solucionará el problema, a menos que esa igualación se haga muy a la baja es decir, convirtiendo los indefinidos en temporales, más que convirtiendo los temporales en indefinidos o buscando un punto intermedio (Cebrian et al, 2011).

En la literatura reciente, sin embargo, hay varias líneas de investigación que resaltan la influencia de la legislación laboral sobre la segmentación del mercado de trabajo. Por ejemplo, por lo que respecta a países en vías de desarrollo, en los que la segmentación se traduce en la existencia de un sector formal, sometido a la legislación laboral, tributaria, etc., y un sector informal, en el que dicha legislación no necesariamente se aplica, Fields (2009) destaca la importancia de un conjunto de factores institucionales, tales como los salarios mínimos, los sindicatos, las políticas de salariales del sector público, las multinacionales y las reglamentaciones laborales para la fijación de salarios, más allá de la interacción entre la oferta y la demanda laboral. En el caso de los países desarrollados, entre los que España destaca como ejemplo paradigmático de segmentación laboral, Bentolila et al (2012) señalan que el origen de los mercado duales, al menos para el caso español, es la interacción de las instituciones, más concretamente de las políticas de protección al empleo y la regulación de la negociación colectiva, que ha exacerbado las diferencias de condiciones laborales entre "insiders" y "outsiders" (distinción que podría asimilarse a la distinción institucionalista entre los trabajadores de los mercados primario y secundario). Trabajos como los de Costain, Jimeno y Thomas (2010); Bentolila, Dolado y Jimeno (2012) y Garcia Perez y Osuna (2012), entre otros, ofrecen argumentos teóricos por los que el cambio de marco institucional hacia un sólo contrato, en el que las únicas diferencias en los costes de despido tendrían que ver con la antigüedad del trabajador en el puesto, reduciría la rotación laboral, lo que generaría un cambio en el comportamiento de los empresarios que con este nuevo contrato se sentirían más seguros a la hora de dar formación a sus trabajadores, que a su vez verían aumentada su productividad laboral. Desde el punto de vista agregado este contrato también supondría una reducción del desempleo, antes shock externos, y por tanto, una reducción de los costes asociados al mismo

Ante estos resultados, Toharia (2010) argumenta que, dado que los trabajadores no constituyen un colectivo homogéneo y que los empresarios saben muy bien a qué trabajadores les interesa formar (a los que ofrecerán contratos en prácticas antes de hacerlos indefinidos o directamente los 
harán indefinidos) y a qué trabajadores no interesa formar, por tratarse de personas fácilmente reemplazables, un aumento de los costes de extinción para los puestos poco cualificados de naturaleza temporal podría inhibir algo la contratación aunque, al tratarse de costes ciertos pueden fácilmente descontarse e incluirse en la negociación salarial (como sucede actualmente con los temporales), pero no mejoraría el rendimiento de las inversiones en formación de las empresas. Una disminución de los costes de extinción para los indefinidos tampoco produciría efectos notables, a la vista de la experiencia reciente que ha demostrado que a las empresas españolas han contratado trabajadores personas con contratos asociados a un mayor coste de extinción, incluso cuando podían hacerles un contrato de fomento de la contratación indefinida, cuyo coste de extinción es menor.

\section{Comentarios finales}

Para nosotros, que aprendimos mucho de las conversaciones con Luis Toharia, aunque en uno de los casos mantuviéramos diferencias importantes con él acerca de las respuestas correctas a las tres preguntas anteriores, es muy triste no poder seguir buscando con él las soluciones al grave problema laboral de nuestro país, especialmente en un contexto como el actual en el que las reformas laborales se ejecutan con tanto desconocimiento.

Muchos de los argumentos utilizados por él y de los resultados que él obtuvo se utilizan profusamente hoy en debates sobre reformas laborales y políticas de empleo, pero es muy desafortunado que quienes lo hacen no llegan a mostrar el grado de comprensión y de capacidad de justificación empírica sobre estas cuestiones que él tenía. Estas cuestiones siguen planteadas $\mathrm{y}$, como en todo proceso científico, seguiremos acumulando conocimiento sobre ellas. Es una verdadera lástima que él no pueda contribuir al mismo, ni disfrutar de él.

\section{Bibliografía}

Albert, C., C. García-Serrano y V. Hernanz (2005), "Firm-Provided Training and Temporary Contracts, Spanish Economic Review, 7, 67-88.

Andrés, J., Boscá, J.E., Doménech, R. y J. Ferri (2009), “Job Creation in Spain: Productivity Growth, Labour Market Reforms or Both?”, Working Paper IEI WP0903. 
Blanchard, O. y A. Landier (2002). "The Perverse Effects Of Partial Labour Market Reform: Fixed-Term Contracts In France," Economic Journal, 112(480),

Bentolila, S., Cahuc, P., Dolado, J. J. y Le Barbanchon, T. (2010) "TwoTier Labor Markets in the Great Recession: France vs. Spain," CEPR Discussion Papers 8152, C.E.P.R. Discussion Papers.

Bentolila, S., Dolado, J. y Jimeno, J.F. (2008), "Two-Tier Employment Protection Reforms: The Spanish Experience", CESifo DICE Report 4/2008

Bentolila, S., Dolado, J. J, y J.F. Jimeno (2012). "Reforming an InsiderOutsider Labor Market: The Spanish Experience," IZA Journal of European Labor Studies.

Boeri, T. y Garibaldi, P. (2007), 'Two-tier Reforms of Employment Protection Legislation: A Honeymoon Effect', Economic Journal 117, 35785.

Bowles, S. y Gintis, E. (1975), "The Problem with Human Capital Theory. A Marxian Critique", American Economic Review, 65(2), 74-82

Cebrián, I. y Toharia, L. (2008), "La entrada en el mercado de trabajo español: un análisis basado en la Muestra Continua de Vidas Laborales", Revista de Economía Aplicada, E-1 (16): 137-172.

Cebrián, I., Moreno, G. y Toharia, L. (2011): "La Estabilidad Laboral y los Programas de Fomento de la Contratación Indefinida", Revista de Hacienda Pública, 198, 103-130.

Costain; J., Jimeno, J.F. y Thomas, C. 2010. "Employment fluctuations in a dual labor market," Working Papers 1013, Banco de España.

Davia, M.A. y Hernanz, V. (2004): "Temporary Work and Segmentation in the Spanish Labour Market: An Empirical Analysis through the Study of Wage Differentials", Spanish Economic Review, volume 6, number 4, pp 291-318

Dolado, J.J., García, C. y Jimeno, J.F. (2002), Drawing Lessons from the Boom of Temporary Jobs in Spain”, Economic Journal. 
Dolado, J.J. y Stucchi, R. (2008) "Do Temprary Contracts Affect TFP? Evidence from Spanish Manufacturing Firms," CEPR Discussion Papers 7055 .

Doringer, P. y Piore, M.J. (1971), Internal Labor Markets and Manpower Analysis, New York, John Wiley (versión española en Ministerio de Trabajo y Seguridad Social, 1985)

García Perez, J.I y Osuna V. (2012) Dual Labour Markets and the Tenure Distribution: Reducing Severance Pay or Introducing a Single Contract? Working Papers FEDEA.

Garrido, L. y Requena, M. (1996), La Emancipación de los jóvenes en. España, Injuve, Madrid

Gutiérrez-Domènech, M. (2008) "The impact of the labour market on the timing of marriage and births in Spain" Journal of Population Economics, 21(1), 83-110.

Hernanz, V. Origo, F., Samek, M. y Toharia, L. (2009) Dreaming of a Stable Job: The Transition of Temporary Workers in Italy and Spain. Edward Elgar.

Hernanz, V. y Toharia, L. (2006), Do Temporary Contracts Increase Work Accidents? A Microeconometric Comparison between Italy and Spain. Labour, 20: 475-504.

Jimeno, J.F. y Toharia, L. (1992), "El mercado de trabajo español en el proceso de convergencia hacia la Unión Económica y Monetaria europea", Papeles de Economía Española, 52-53: 78-107.

Jimeno, J.F. y Toharia, L. (1994), Unemployment and Labour Market Flexibility: the case of Spain, Geneva, International Labour Office.

Jimeno, J.F. and Toharia, L. (1996), "Effort, Absenteeism, and Fixed Term Employment Contracts". Revista Española de Economía, 13(1): 105-119.

Piore, M.J. y Berger, S. (1980), Dualism and discontinuity in industrial societies, Cambridge, Cambridge University Press.

Madera, R. (2012) Dual Labor Markets and Productivity. Master Thesis CEMFI No. 1201. January 2012. CEMFI. 
Saint-Paul, G. (1996): Dual Labor Markets - A Macroeconomic Perspective. MIT Press, Cambridge, MA.

Segura, J., Durán, F., Toharia, L. y Bentolila, S. (1991), Análisis de la contratación temporal en España, Madrid, Ministerio de Trabajo y Seguridad Social.

Toharia, L. (1981), "Un test histórico de la teoría de la eficiencia de los mercados internos de trabajo", Cuadernos de Economía, 9-25, 355-380.

Toharia, L (1983) El mercado de trabajo: teorías y aplicaciones. Alianza Editorial

Toharia, L. (1996), "Empleo y paro en España: evolución, situación y perspectivas", Ekonomiaz, 35(2), 35-67.

Toharia, L. (dir) (1998), El mercado de trabajo en España, Madrid, McGraw-Hill

Toharia, L. (dir.) (2005), El problema de la temporalidad en España: un diagnóstico, Madrid, Ministerio de Trabajo y Asuntos Sociales.

Toharia (2009), "Las reformas necesarias para la economía española: el mercado de trabajo". Mimeo.

Toharia, L., Albert, C., García-Serrano, C., Malo, M.A., Davia, M.A. y Arranz, J.M. (2007), Empleo e inclusión social. Ministerio de Trabajo y Asuntos Sociales, Madrid.

Toharia, L., Davia, M. A. y Hernanz, V. (2001) Flexibilidad, juventud y trayectorias laborales en el mercado de trabajo español, Centro de Investigación Sociológicas (CIS), Madrid.

Williamson, O., M. Wachter y J. Harris (1975), 'Understanding the employment relation', The Bell Journal of Economics, 6, 250-78. 